\title{
Obesity-associated family with sequence similarity 13 , member $A$ (FAM13A) is dispensable for adipose development and insulin sensitivity
}

\author{
Jiazhen Tang ${ }^{1,2} \cdot$ Hongyi Zhou $^{2} \cdot$ Khushboo Sahay $^{2} \cdot$ Wenqiong Xu ${ }^{1,2} \cdot$ Jing Yang ${ }^{3} \cdot$ Wei Zhang ${ }^{4} \cdot$ Weiqin Chen $^{2}$
}

Received: 8 March 2018 / Revised: 22 August 2018 / Accepted: 2 September 2018 / Published online: 9 October 2018

(c) The Author(s) 2018. This article is published with open access

\begin{abstract}
Background Obesity and its associated morbidities represent the major and most rapidly expanding world-wide health epidemic. Recent genome-wide association studies (GWAS) reveal that single nucleotide polymorphism (SNP) variant in the Family with Sequence Similarity 13, Member A (FAM13A) gene is strongly associated with waist-hip ratio (WHR) with adjustment for body mass index (BMI) (WHRadjBMI). However, the function of FAM13A in adipose development and obesity remains largely uncharacterized.

Methods The expression of FAM13A in adipose tissue depots were investigated using lean, genetic obese and high fat dietinduced obese (DIO) animal models and during adipocyte differentiation. Stromal vascular cells (SVCs) or 3T3-L1 cells with gain and loss of function of FAM13A were used to determine the involvement of FAM13A in regulating adipocyte differentiation. Adipose development and metabolic homeostasis in $\mathrm{Faml} 3 \mathrm{a}^{-/-}$mice were characterized under normal chow and high fat diet feeding.

Results Murine FAM13A expression was nutritionally regulated and dramatically reduced in epididymal and subcutaneous fat in genetic and diet-induced obesity. Its expression was enriched in mature adipocytes and significantly upregulated during murine and human adipogenesis potentially through a peroxisome proliferator-activated receptor-gamma (PPAR $\gamma$ )-dependent mechanism. However, Fam $13 a^{-/-}$mice only exhibited a tendency of higher adiposity and were not protected from DIO and insulin resistance. While Fam $13 a^{-1-}$ SVCs maintained normal adipogenesis, overexpression of FAM13A in 3T3-L1 preadipocytes downregulated $\beta$-catenin signaling and rendered preadipocytes more susceptible to apoptosis. Moreover, FAM13A overexpression largely blocked adipogenesis induced by a standard hormone cocktail, but adipogenesis can be partially rescued by the addition of PPAR $\gamma$ agonist pioglitazone at an early stage of differentiation.

Conclusions Our results suggest that FAM13A is dispensable for adipose development and insulin sensitivity. Yet the expression of FAM13A needs to be tightly controlled in adipose precursor cells for their proper survival and downstream adipogenesis. These data provide novel insights into the link between FAM13A and obesity.
\end{abstract}

Electronic supplementary material The online version of this article (https://doi.org/10.1038/s41366-018-0222-y) contains supplementary material, which is available to authorized users.

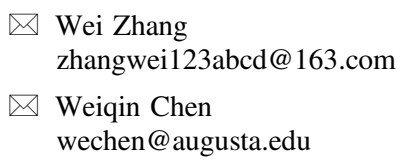

Wei Zhang

zhangwei123abcd@163.com

$\triangle$ Weiqin Chen

wechen@augusta.edu

1 Department of Endocrinology and Metabolism, The First Affiliated Hospital of Nanchang University, Nanchang, Jiangxi Province 330006, China

\section{Introduction}

Obesity and its associated health comorbidities is a worldwide epidemic which imposes a serious economic and health burden on society. Genetic factors have been attributed to around $40-70 \%$ of inter-individual variability in

2 Department of Physiology, Medical College of Georgia at Augusta University, Augusta, GA 30912, USA

3 Department of Comparative Biosciences, University of Illinois at Urbana-Champaign, Urbana, IL 61802, USA

4 Department of Respiratory Medicine, The First Affiliated Hospital of Nanchang University, Nanchang, Jiangxi Province 330006, China 
body mass index (BMI) which is commonly used to assess overall obesity. Overall adiposity and body fat distribution are both heritable traits and well-established predictors of adverse metabolic outcomes [1, 2]. Recent genome-wide association studies (GWAS) have successfully identified a large number of obesity-associated genetic loci. Among these, 97 gene variants are associated with overall adiposity measured by body mass index (BMI) [3] and 49 gene variants are associated with fat distribution measured by waist circumference (WC) and waist to hip ratio (WHR) [4]. However, the majority of these variants have not been characterized. Their biological functions and pathological relevance to adipose biology and obesity remain unknown.

A recent GWAS study with the largest cohort identified a genetic variant (rs9991328) in the Family with Sequence Similarity 13, Member A (FAM13A) gene to be highly associated with WHR adjusted BMI (WHRadjBMI) [4], suggesting FAM13A may play a role in body fat distribution.FAM13A expression was abundant in human adipose tissue [5]. The expression of FAM13A gene was shown to be associated with adipose morphology, specifically adipocyte cell numbers (hyperplasia) [6]. Single nucleotide polymorphisms (SNPs) in FAM13A have been widely associated with chronic obstructive pulmonary disease (COPD) [7-9], asthma severity [10], lung cancer [11] as well as pulmonary fibrosis [12], underscoring an important involvement of FAM13A in human lung disease etiology. The COPD risk allele at the FAM13A locus was shown to be associated with increased expression of FAM13A in human lung samples [13]. The biological function of FAM13A in lung physiology remains elusive. FAM13A was found to form complex with protein phosphatase $2 \mathrm{~A}$ and $\beta$-catenin, and exhibited different effects on the stability of $\beta$-catenin in different experimental settings, suggesting that FAM13A is likely a context-dependent regulator of the Wnt pathway [13, 14]. Most recently, FAM13A was identified to promote fatty acid oxidation (FAO) possibly by interacting with and activating Sirtuin 1 (Sirt1), thereby increasing the expression of CPT1 $\alpha$ as well as other $\beta$-oxidation genes in lung [15]. Despite its association with fat distribution, to date, no study has explored the potential role of FAM13A in regulating adipogenesis and obesity. In this study, we investigated the expression and function of FAM13A in adipose development and metabolic homeostasis in vitro and in vivo.

\section{Materials and methods}

\section{Animal experiments}

All animal procedures were approved by Augusta University's Institutional Animal Care and Use Committee
(IACUC). Mice were maintained under standard conditions with controlled $12 \mathrm{~h} / 12 \mathrm{~h}$-light-dark cycle and $21 \pm 1{ }^{\circ} \mathrm{C}$ room temperature. Lep ${ }^{o b / o b}, L_{e p r} r^{d b / d b}$ and C57BL/6 J mice were obtained from Jackson Laboratory (Bar Harbor, ME). $\mathrm{Fam}_{13 a^{-/}}$mice were backcrossed to C57BL/6 (Stock\#: 027, Charles River) for seven generations and genotyped as previously reported [14]. 4-5 week old $\mathrm{Faml}_{3} \mathrm{a}^{+/+}$and $\mathrm{Fam}_{13 a^{-1}}$ littermates were fed with high fat diet (HFD, D12492, 60\% Kcal from fat, Research Diets, NJ) for up to 12 weeks. Mice were not randomized. Six week old C57BL/6 J male mice were simply randomized into two experimental groups for ad libitum access to low fat control diet (LFD, D12450B, 10\% Kcal from fat, Research Diets, $\mathrm{NJ}$ ) and $60 \%$ HFD for up to 12 weeks as reported [16]. All mice studies were not blinded. All mice were sacrificed after $4 \mathrm{~h}$ fasting with isoflurane.

\section{Human tissues}

Human adipose tissues were collected from obese (BMI > 40) patients undergoing gastric bypass surgeries. The study protocol was approved by the Institutional Review Boards of the Medical College of Georgia at Augusta University and informed consent was obtained from the subjects.

\section{Cell culture, plasmids, transient transfection, luciferase assay}

All cell culture reagents were obtained from Fisher Scientific Inc. (Pittsburgh, PA). Insulin, dexamethasone, isobutylmethylxanthine (IBMX), puromycin were obtained from Sigma-Aldrich (St. Louis, MO). The 3T3-L1 (ATCC CL-173) and NIH/3T3 cells (ATCC CRL-1658) were cultured following ATCC instructions. pLightSwitch-Luc vector harboring predicted murine Faml3a promoter was obtained from Active Motif (Carlsbad, CA). pSV Sport PPAR 22 was obtained from Addgene (\#8862). Promoterdriven luciferase activities were analyzed in NIH/3T3 cells by using LightSwitch ${ }^{\mathrm{TM}}$ Luciferase Assay System with or without PPAR $\gamma 2$ overexpression.

\section{Retrovirus and lentivirus production and infection}

pBABE-puro-PPAR gamma2 vector was obtained from Addgene (\#8859). Retroviral packaging Bosc-23 cells were cotransfected with the targeting plasmid and packaging vector pCL-eco (Imgenex, Sorrento Valley, CA). Lentiviral particles were produced by transient transfection of 293 $\mathrm{T}$ cells with a packaging plasmid psPAX2 and envelope plasmid pMD2.G together with pSin-EF2-Puro vector alone or pSin-EF2-FAM13A-FLAG-Puro. $48 \mathrm{~h}$ after transfection, the culture media containing the virus particles were collected and mixed with DMEM 10\% FBS at 1:1 to infect 
3T3-L1 preadipocytes or NIH-3T3 fibroblasts in the presence of $8 \mu \mathrm{g} / \mathrm{ml}$ polybrene. Cells were then selected with $2 \mu \mathrm{g} / \mathrm{ml}$ puromycin for at least 4 days before analysis or differentiation.

\section{MTT assay, Edu proliferation and immunofluorescence microscopy}

Cell number was determined by Vybrant MTT cell proliferation assay kit (Invitrogen, Grand Island, NY) following manufacturer's instruction. Immunofluorescence staining against FAM13A was performed as previously described [17]. For proliferation, same amount of cells were plated and grown on coverslips. Cells were labeled with Edu for $24 \mathrm{~h}$ by Click-iT ${ }^{\mathrm{TM}}$ EdU Alexa Fluor ${ }^{\mathrm{TM}} 488$ Imaging Kit (ThermoFisher Scientific) as instructed. Cells were then counter-stained with Dapi and analyzed using fluorescent microscope. Cells from six random fields were counted for each treatment and the proliferation rate was defined as (no. of fluorescein-labeled nuclei)/(no. of Dapi nuclei) $\times 100$.

\section{FACS analysis of apoptosis}

Cells with and without serum starvation for $24 \mathrm{~h}$ were fixed and permeabilized. Cells were then stained with APO-BrdU TUNEL Assay Kit with Alexa Fluor 488 anti-Brdu (A23210, ThermoFisher Scientific) and LIVE/DEAD Fixable for Far Red Dead Cell Stain Kit, for 633 nm excitation (L34973, ThermoFisher Scientific). Apoptosis was assessed by FACS analysis using FACSCalibur (Becton Dickinson) under $488 \mathrm{~nm}$ and $635 \mathrm{~nm}$ lasers. Cells negative for both far-red dye and TUNEL staining are live cells; far-red-dye negative, TUNEL-positive staining cells are early apoptotic cells; far-red-dye positive, TUNEL-negative staining cells are dead cells; far-red-dye positive, TUNEL-positive staining cells are primarily cells in late stages of apoptosis. The total percentage of early and late apoptotic cells were compared.

\section{Adipose tissue fractionation, adipocyte differentiation, triacylglycerol (TAG) content measurement and oil-red 0 (ORO) staining}

Mouse or human adipose tissues were digested with collagenase (Sigma Aldrich), filtered, and centrifuged to separate mature floating adipocytes from the pelleted stromal vascular fraction (SVF) as described previously [18]. Preadipocytes rich SVF pellets and 3T3-L1 cells were cultured to confluence and induced to differentiate into mature adipocytes by addition of insulin, dexamethasone, and IBMX (DMI) as previously described [18]. At indicated days, cells were washed and directly dissolved in PBS containing $1 \%$ triton $\mathrm{X}-100$. The intracellular TAG in the adipocytes was measured with an Infinity Triglyceride assay kit (ThermoFisher Scientific). The TAG content was normalized to the amount of cellular protein as determined using a Bradford protein assay (Bio-Rad) and expressed as per milligram of protein. ORO staining was performed and photographed with camera or under microscopy as previously described [18].

\section{Plasma biochemistry}

Plasma nonesterified fatty acid (NEFA) (Wako Chemicals USA Inc.), glycerol (Sigma-Aldrich), total cholesterol, total triacylglycerol levels (Fisher Scientific Inc.) were measured colorimetrically.

\section{Histology, body composition, glucose tolerance and insulin tolerance tests}

Tissue samples were fixed, processed, and stained with hematoxylin and eosin (H\&E) and visualized under microscopy. Fat and lean body masses were measured by NMR-based Bruker minispec LF90II (Bruker company, German). Insulin tolerance tests were performed in mice fasted for $6 \mathrm{~h}$ and then injected intraperitoneally (i.p.) with human insulin (Humulin; Novo Nordisk) at $0.75 \mathrm{U} / \mathrm{kg}$ for mice on normal chow diet, and $2.5 \mathrm{U} / \mathrm{kg}$ for mice on HFD. Glucose tolerance tests were performed in $6 \mathrm{~h}$ fasted chow-fed mice injected by i.p. or $16 \mathrm{~h}$ fasted HFD-fed mice administered by gastric gavage with glucose $(1.5 \mathrm{~g} / \mathrm{kg}$ body weight). Blood glucose levels were measured by One-touch Ultra glucose meter before and at 15, 30, 60, and $120 \mathrm{~min}$ after administration.

\section{RNA isolation and real-time quantitative PCR}

Total RNA was isolated from tissues or cells with TRIzol (Invitrogen) and reverse transcribed using MLV-V reverse transcriptase with random primers (Invitrogen). Real-time quantitative RT-PCR was performed on a Stratagene MX3005. Data were normalized to two housekeeping genes (Ppia and 36B4) based on Genorm algorithm and expressed as fold changes relative to control cells or tissues.

\section{Immunoblot analysis}

Tissues and cells were homogenized and lysed in lysis buffer as previously described [19]. The protein concentration was determined by Bradford protein assay (Bio-Rad). Equal amounts of proteins were loaded and immunoblot analysis was carried out according to standard protocol. The blots were visualized using the ECL chemiluminescence system by Amersham Imager 600 
(GE healthcare) and quantified by densitometric analysis using ImageQuantTL (GE healthcare). The following antibodies were used: rabbit polyclonal antibodies against FAM13A (HPA038109; Sigma Life Science); PPAR $\gamma$ (2435), $\beta$-Catenin (8480), IRS1 (2382), GSK3 $\beta$ (12456), pGSK3 $^{\text {S9 }}$ (9336), SIRT1 (9475) from Cell Signaling Technology; GAPDH (60004-1-IG, Proteintech); PLIN1 (GP29; Progen Biotechnik GmbH); ATGL (10006409; Cayman Chemical); C/EBP $\alpha$ (sc-61, Santa Cruz); $\beta$-ACTIN (MAB1501) from Millipore.

\section{Statistical analysis}

All in vitro experiments were performed in triplicate for at least three times. Animal experiments were performed in at least two independent cohorts. Detailed sample sizes for animal studies were provided in each figure legend. Sample sizes were chosen by power analysis based on pilot studies. Statistical analyses of the data were analyzed by SigmaPlot 13 (Systat Software, Inc) using a two-tailed unpaired $t$ test with equal variance. Data were presented as the mean $\pm \mathrm{SEM}$ with statistical significance set at a $P$ value of $<0.05$.

\section{Results}

\section{FAM13A is nutritionally regulated and downregulated in genetic and diet-induced obesity}

The significant association of SNPs near the FAM13A gene locus with WHRadjBMI suggests FAM13A may play a role in regulating adipose tissue distribution. We first examined the expression of FAM13A in adipose tissue of murine obese models. Surprisingly, we found FAM13A expression in epididymal white adipose tissue (eWAT) of genetic obese $L e p^{o b / o b}$ and $L e p r^{d b / d b}$ mice were dramatically downregulated at both mRNA and protein levels as compared to their lean counterparts (Fig. 1a, b). Likewise, the mRNA and protein expression of FAM13A in eWAT and inguinal subcutaneous WAT (sWAT) were markedly reduced in our previously reported HFD-induced obese
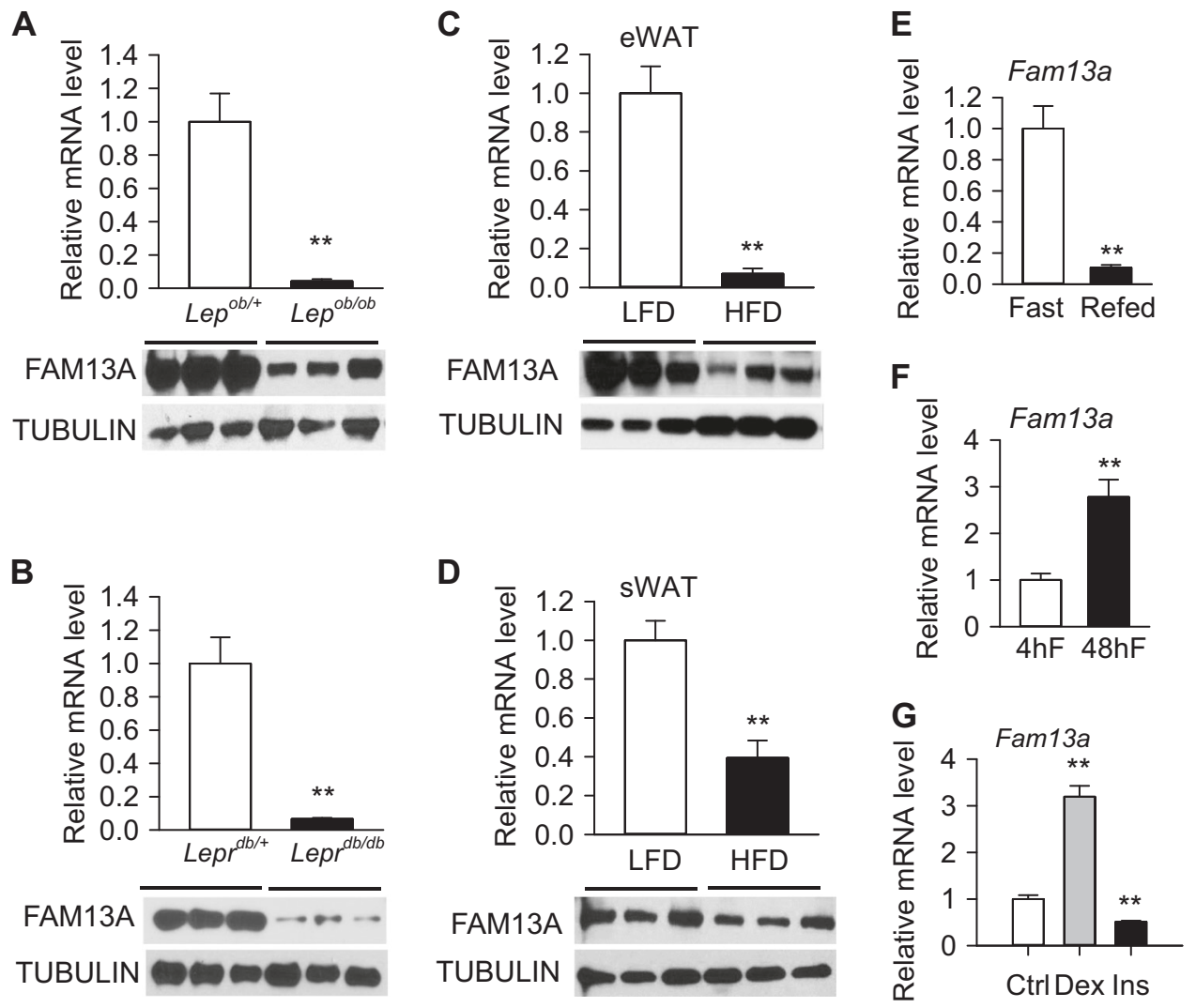

Fig. 1 FAM13A is nutritionally regulated and downregulated in genetic and diet-induced obesity. $\mathbf{a}, \mathbf{b}$ Gene $(n=6 /$ group $)$ and protein expression ( $n=3$ /group) of FAM13A in epididymal white adipose tissue (eWAT) of 3 months old male genetic obese (a) Lep $^{\text {ob/ob }}$ and (b) Lepr ${ }^{d b / d b}$ mice as compared to their lean countparts. c, d Gene ( $n=10$ /group) and protein ( $n=3$ /group) expression of Fam13a in (c) eWAT and (d) inguinal subcutaneous WAT (sWAT) of low fat diet (LFD) and high fat diet (HFD)-fed male C57BL/6 J mice. e, f mRNA expression of Fam13a in eWAT of 10 weeks old male C57BL/6 J mice after (e) a $18 \mathrm{~h}$ fast and $6 \mathrm{~h}$ refeed; (f) $4 \mathrm{~h}$ fast ( $4 \mathrm{hF})$ as compared to $48 \mathrm{~h}$ prolonged fasting $(48 \mathrm{hF})(n=7 /$ group). $\mathbf{g}$ mRNA expression of Fam13a in mature 3T3-L1 adipocytes after $18 \mathrm{~h}$ treatment with dexamethasone (Dex) and insulin (Ins) as compared to vehicle-treated control (Ctrl) cells. Representative data were shown from 3 independent experiments in triplicate. $* * p<0.005$ 
mouse model [16] as compared to LFD-fed lean mice (Fig. 1c, d). These data underscore a dramatic decrease of FAM13A in obese adipose tissue.

Given the significant downregulation of FAM13A in adipose tissue of animal models with hyperphagia, we then asked whether FAM13A is nutritionally regulated. We found that Fam 13 a mRNA was markedly reduced in eWAT of $6 \mathrm{~h}$ refed C57BL/6 J mice as compared to overnight $(18 \mathrm{~h})$ fasted mice (Fig. 1e). Conversely, Fam13a transcript was upregulated ( 3 -fold) in eWAT of C57BL/6 J mice after prolonged $48 \mathrm{~h}$ food deprivation as compared to mice after a $4 \mathrm{~h}$ fast (Fig. 1f). To further confirm Fam13a gene is a nutritionally responsive gene in adipose tissue, we treated 3T3-L1 mature adipocytes with dexamethasone and insulin to mimic fasting and refeeding responses. We found Fam13a was upregulated by catabolic hormone dexamethasone but inhibited by insulin treatment (Fig. 1g), similar to a well-known catabolic gene adipose triglyceride lipase (ATGL) [20]. Collectively, these data demonstrate that Fam $13 a$ expression in adipose tissue is dynamically regulated in response to changes in the nutritional status.

\section{FAM13A is upregulated during adipocyte differentiation potentially through PPARY}

To further analyze the role of FAM13A in adipose biology, we first studied the expression of FAM13A during adipocyte differentiation of 3T3-L1, a well-established adipocyte differentiation model. At both mRNA and protein levels, FAM13A was gradually upregulated during the course of preadipocyte differentiation into mature adipocytes and reached the highest level in mature adipocytes (Fig. 2a, b). The expression of $A p 2$ and PPAR $\gamma$ were used as markers for adipogenesis. Similarly, we found that Faml3a gene expression was upregulated during adipocyte differentiation of murine primary stromal vascular cells (SVCs) (Fig. 2c). Its expression was also highly enriched in fractionated adipocytes as compared to stromal vascular fractions (SVF) (Fig. 2d). These data clearly suggest that FAM13A exhibits an adipocyte-specific expression signature.

To identify the potential mechanisms underlying FAM13A upregulation during adipogenesis, we next generated stable NIH-3T3 cells overexpressing PPAR 2 , the master transcription factor governing adipocyte differentiation. Fam 13 a mRNA expression was elevated $\sim 14$-fold in NIH-3T3 cells stably transduced with pBABEpuroPPAR 2 retrovirus as compared to vector infected cells (Fig. 2e). Western blot confirmed the overexpression of PPAR $\gamma 2$ and the induction of FAM13A protein (Fig. 2f). Furthermore, we performed a luciferase assay in NIH/3T3 cells using pLight-Switch murine Fam13a-pro, a luciferase reporter driven by Fam $13 a$ promoter. Overexpression of PPAR $\gamma 2$ significantly upregulated Fam $13 a$ promoter activity (Fig. 2g). These data suggest that PPAR $\gamma 2$ is upstream of FAM13A expression. Moreover, FAM13A gene expression was also markedly upregulated during human preadipocyte differentiation into mature adipocytes (Fig. 2h) and enriched in mature adipocyte fraction as compared to SVF isolated from human subcutaneous adipose tissue (Fig. 2i). Taken together, we conclude that FAM13A is regulated by the master adipogenic transcription factor PPAR $\gamma$, and its expression is gradually increased during murine and human adipocyte differentiation.

\section{Loss of FAM13A minimally affects adiposity, diet- induced obesity and metabolic homeostasis}

To dissect roles of FAM13A in regulating adipose tissue development and distribution, we first analyzed the protein expression of FAM13A in lung, liver, and various adipose depots. We observed that the expression levels of FAM13A in eWAT, sWAT and brown adipose tissue (BAT) were much higher as compared to that in lung; while no FAM13A was detected in liver (Fig. 3a). As expected, FAM13A was not detectable in tissues from Faml3a $a^{-/-}$mice (Fig. 3a). Body weight, circulating levels of plasma TAG, cholesterol, NEFA and glycerol were comparable between the wild type and Fam $13 a^{-/-}$male and female mice (Table S1). Fat mass and lean mass were also similar between 16 week old $\mathrm{Faml}_{3} \mathrm{a}^{+/+}$ and $\mathrm{Faml}_{3} \mathrm{a}^{-/-}$mice (Fig. 3b). After dissection, Faml3a ${ }^{-/-}$ mice did demonstrate a tendency towards higher masses of eWAT and sWAT as compared to Fam $13 a^{+/+}$mice (Fig. 3c), but maintained normal histological appearance of eWAT, sWAT, BAT and liver (Fig. 3d). Western blot confirmed similar expression of adipose tissue marker proteins (PPAR $\gamma$ and PLIN1), further suggesting lack of overt difference in WAT of $\mathrm{Faml3a}^{-1-}$ mice (Fig. 3e). Consistent with this,

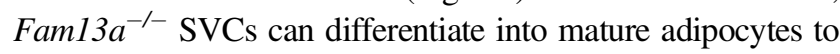
a similar degree as its wild-type counterparts, evidenced by comparable mRNA expression of PPAR $\gamma$ and Ap2 at D0 and D8 adipocytes (Fig. S1a), similar protein expression of PPAR $\gamma$ and PLIN1 in D8 mature adipocytes (Fig. S1b), as well as ORO stained neutral lipids (Fig. S1c). Besides, Fam $13 a^{-/-}$mice also displayed no obvious perturbations in whole body glucose tolerance and insulin sensitivity (Fig. 3f, $\mathrm{g}$, respectively). Notably, lack of overt changes in Faml $3 a^{-/-}$ adipocytes and mice was not due to compensatory upregulation of its known paralog genes (Faml3b and Faml3c) (Fig. S1b and Fig. S2f respectively). These data indicate despite the abundant expression of FAM13A in mature adipocytes, loss of FAM13A exerts little impact on adipocyte differentiation, adiposity and metabolic homeostasis.

When subjected to high fat diet, Fam $13 a^{-/-}$mice retained similar body weight gain (Fig. S2a) and fat composition (Fig. S2b). There was a slight (19\%) increase in sWAT mass in Fam $13 a^{-/-}$mice as compared to Faml3a $a^{+/+}$ 

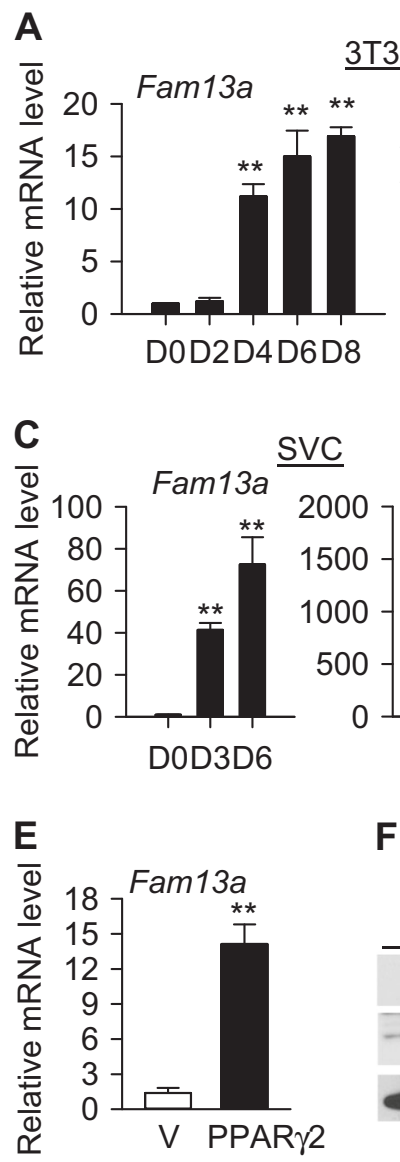

$\mathbf{F}$

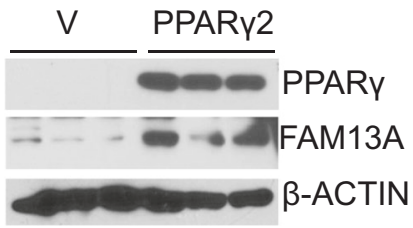

B

Differentiation days

$\begin{array}{lllll}0 & 2 & 4 & 6 & 8\end{array}$

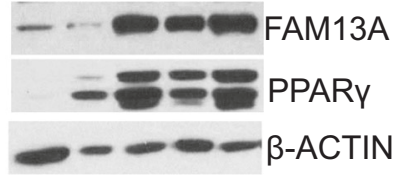

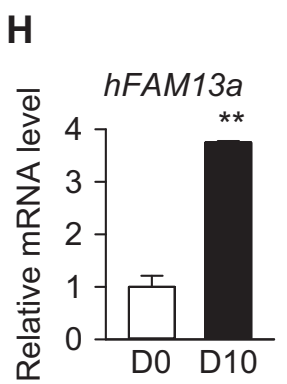

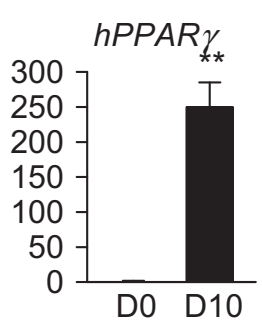

Fig. 2 Fam13a is upregulated during adipocyte differentiation potentially through PPAR $\gamma$. a, b Time course of Fam13a (a) mRNA and (b) protein expression during induced differentiation of 3T3-L1 cells by DMI. The expression of Ap2 mRNA and PPAR $\gamma$ protein was used as positive controls. c Time course of Fam13a and Plin1 mRNA expression during induced differentiation of mouse primary stromal vascular cells (SVCs) by DMI. ** $p<0.005$ vs D0. d mRNA expression of Fam13a and Plin1 in stromal vascular fraction (SVF) and adipocytes (Adipo) fractionated from eWAT of 10 week old male C57BL/6 J mice $(n=6)$. e, $\mathbf{f}$ mRNA $(\mathbf{e})$ and protein expression $(\mathbf{f})$ of FAM13A in NIH/3T3 cells stably transduced with empty lentiviruses

mice (Fig. 3h). However, no significant difference in adipocyte sizes was detected between mutant and control animals (Fig. S2c), and Faml3a ${ }^{-1-}$ mice also maintained similar glucose tolerance (Fig. 3i) and insulin resistance
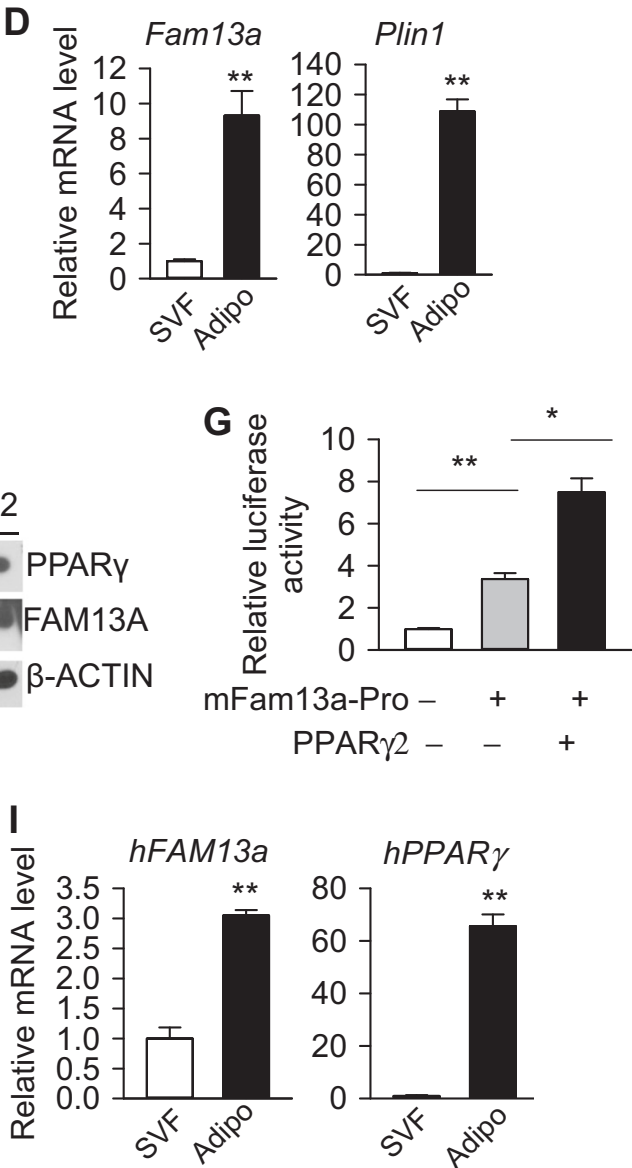

(V) or lentivirus overexpressing PPAR $\gamma 2$. g The pLight-switch luciferase reporter construct harboring predicted murine Fam13a promoter were transfected into NIH/3T3 cells together with PPAR $\gamma 2.48 \mathrm{~h}$ after transfection, cells were harvested for luciferase assays. Reporter activity is expressed relative to the transfection with empty plasmid (set to 1). Representative data from 3 independent experiments were shown. h, i mRNA expression of FAM13A and PPAR 2 (h) during induced differentiation of human primary stromal vascular cells and (i) in SVF and adipocytes fractions from sWAT of human adipose tissue. $* p<0.05 ; * * p<0.005$

(Fig. 3j) as compared to their wild-type counterparts under HFD. These data further suggest a minimal effect of FAM13A deficiency in adipose tissue distribution and whole-body insulin action. 
A

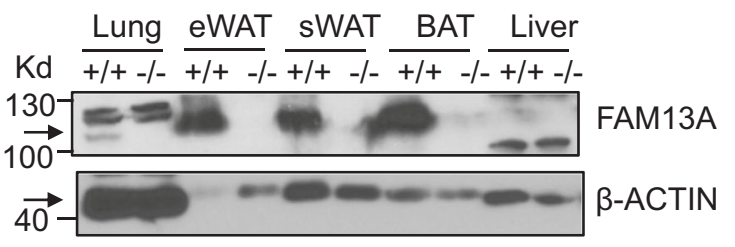

C

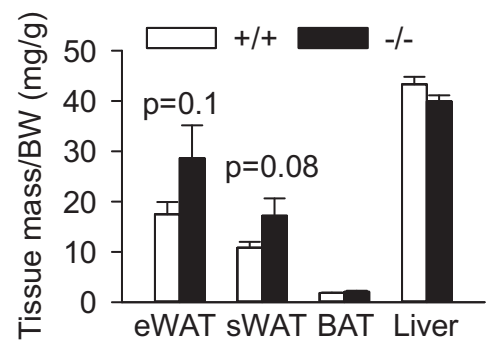

B

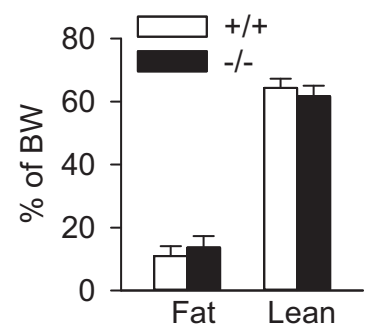

E
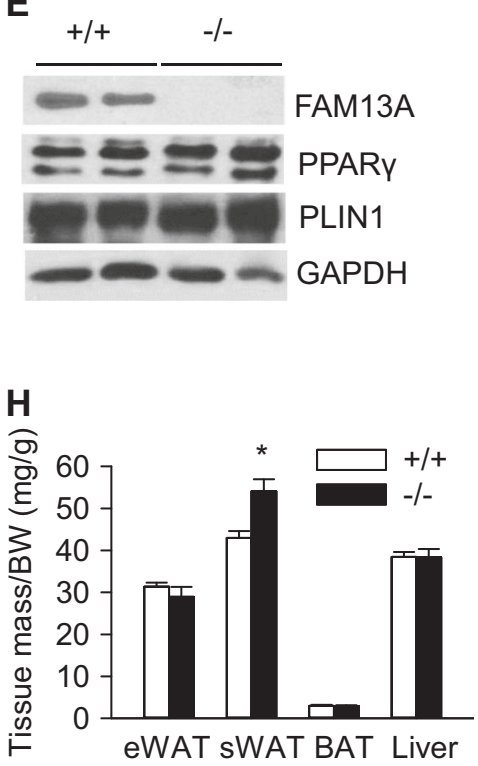

Fig. 3 Loss of Fam13a minimally affects adiposity, diet-induced obesity and metabolic homeostasis in vivo. a FAM13A protein expression in various murine tissues. $\mathbf{b}$ Fat and lean masses presented as \% of body weight. c Tissue masses $(\mathrm{mg})$ as normalized to BW (g). $n=6-7 /$ group. d Representative H\&E images of eWAT, sWAT, BAT and liver. Scale bar, $100 \mu \mathrm{m}$. e Representative western blot of FAM13A and adipose markers PPAR $\gamma$ and PLIN1 in eWAT, $n=4 /$

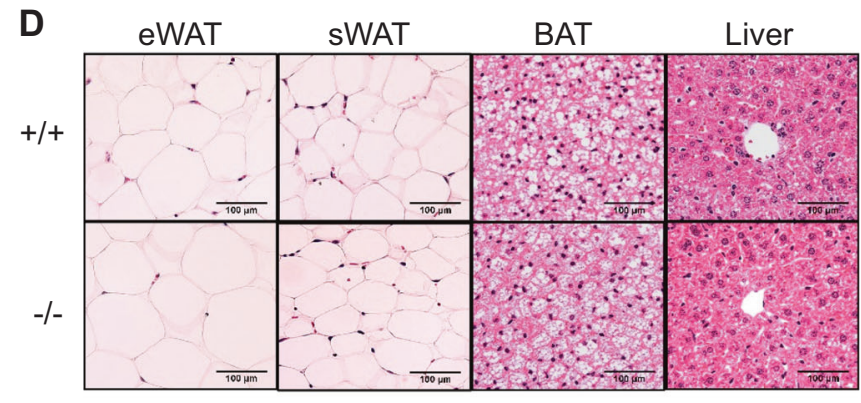

$\mathbf{F}$
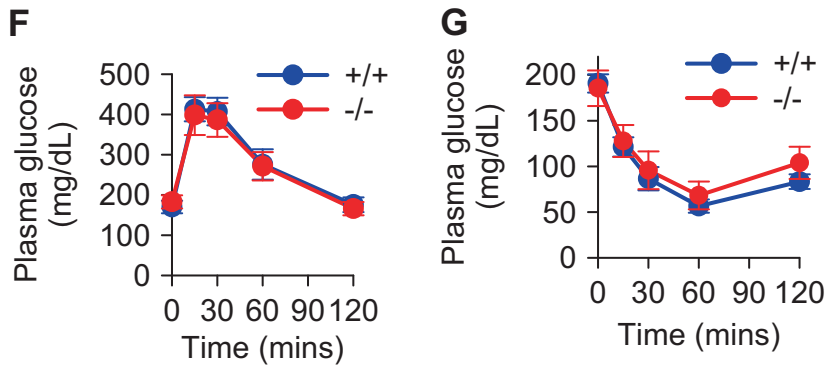

I

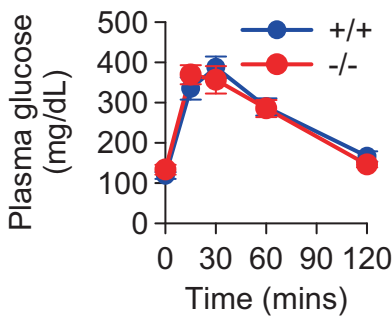

$\mathbf{J}$

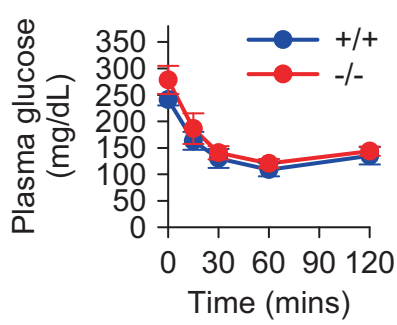

group. f, $\mathbf{g}$ Glucose tolerance test $(\mathbf{f})$ and $(\mathbf{g})$ insulin tolerance test. Chow fed 14-15 week old male Fam $13 a^{+/+}(+/+)$and Faml3a ${ }^{-1-}$ $(-/-)$ mice were used for experiments performed in a. $\mathbf{g} n=6-7 /$ group. h Tissue masses (mg) as normalized to BW (g) after 20 weeks of HFD feeding. $n=5-7 /$ group. i, $\mathbf{j}$ Glucose tolerance test (i) and insulin tolerance test (j) on mice fed with HFD for 11 weeks and 13 weeks respectively ( $n=6-7$ per group)

\section{Overexpression of FAM13A suppresses preadipocyte survival through repressing $\beta$-catenin signaling}

To further dissect the function of FAM13A in adipogenesis, we next generated 3T3-L1 preadipocytes stably overexpressing a full-length $117 \mathrm{kD}$ murine FAM13A and confirmed its overexpression by both immunofluorescence
(Fig. 4a) and western blot (Fig. 4b). Interestingly, the expression of $\beta$-catenin protein (Figs $4 \mathrm{~b}, \mathrm{c}$ ), but not its encoded gene Ctnnb1 (Fig. 4d) was reduced in FAM13Aoverexpressing (OE) preadipocytes as compared to control (vector)-transduced cells. There was a tendency of lower mRNA expression of Wnt1-inducible-signaling pathway protein 2 (Wisp2), frequently used as a marker of canonical 
A

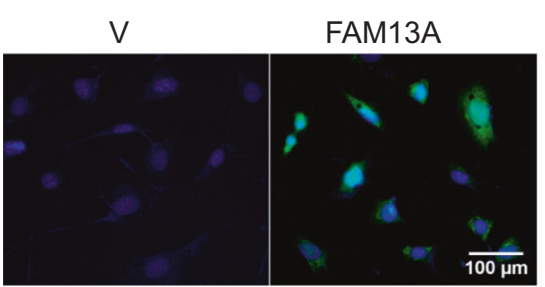

B

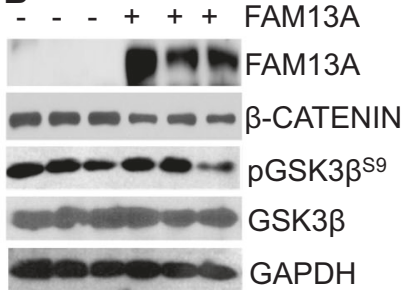

C

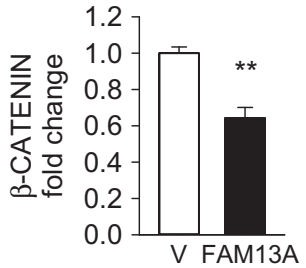

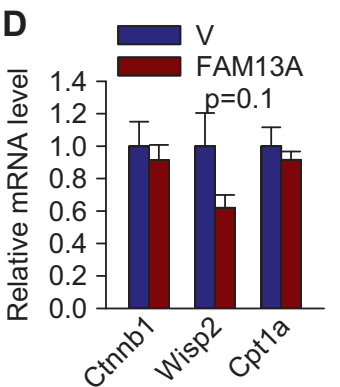

E

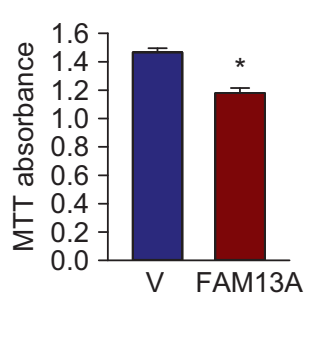

$\mathbf{F}$

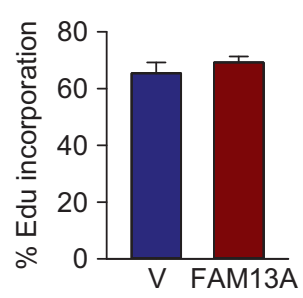

H

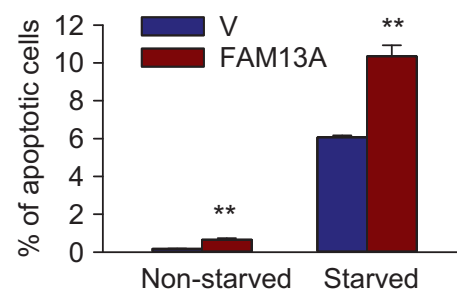

G

Non-starved
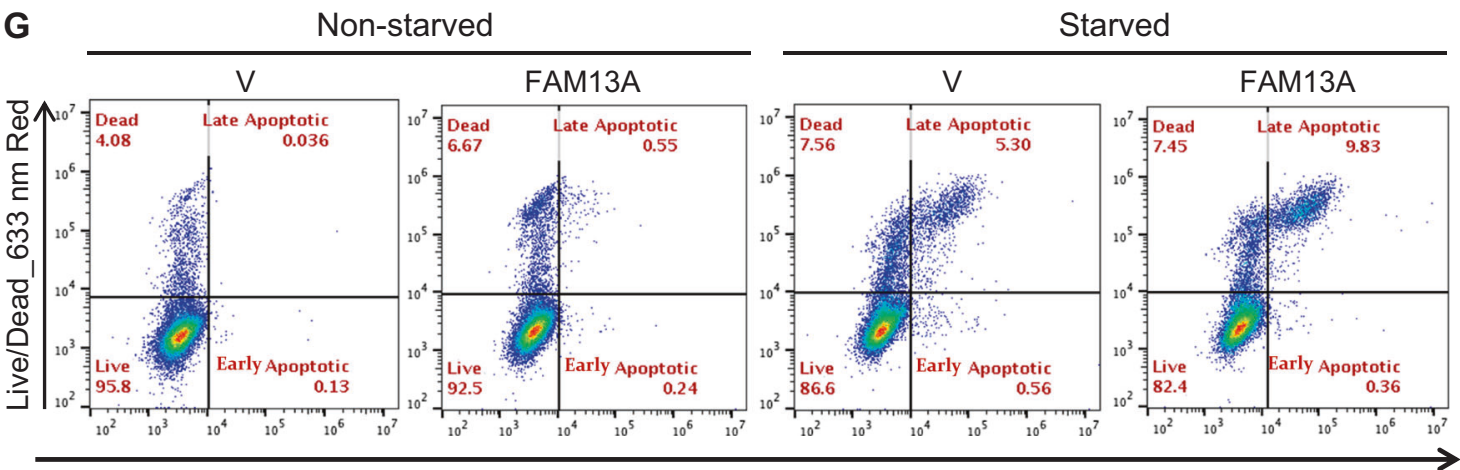

TUNEL_488 nm Green

Fig. 4 Overexpression of murine FAM13A impairs preadipocyte survival. a FAM13A immunofluorescent staining, scale bar, $100 \mu \mathrm{m}$. b, c western blot (b) and quantification (c) of $\beta$-catenin expression. $\mathbf{d}$ qPCR analysis. e MTT viability assay. $\mathbf{f}$ Edu incorporation in vector (V) and FAM13A-overexpressing lentivirus transduced 3T3-L1 preadipocytes. g The levels of apoptosis evaluated by FACS analysis following APO-
BrdU TUNEL staining combined with LIVE/DEAD cell staining in vector $(\mathrm{V})$ and FAM13A-overexpressing lentivirus transduced 3T3-L1 preadipocytes with or without $24 \mathrm{~h}$ serum starvation. The picture shows one of the three experiments. $\mathbf{h}$ Numerical results of early and late apoptotic cells from FACS analysis. Values are presented as mean \pm SEM in 3 separate experiments $* p<0.05 ; * * p<0.005$ vs V
Wnt activation [21] in FAM13A-OE preadipocytes (Fig. 4d), supporting reduced $\beta$-catenin signaling. The canonical Wnt pathway is essential for the survival of adipose precursors by regulating IGF1 expression [22]. Indeed, following plating of equivalent numbers of lentivirally-transduced 3T3-L1 preadipocytes, FAM13AOE preadipocytes displayed lower cell numbers based on MTT assay (Fig. 4e). There was no difference in cell proliferation between vector and FAM13A-OE preadipocytes (Fig. 4f). However, FAM13A-OE 3T3-L1 preadipocytes were more susceptible to apoptosis which was further exacerbated after serum starvation (Fig. $4 \mathrm{~g}, \mathrm{~h}$ ). These data underscore a pro-apoptotic act of FAM13A potentially through downregulating $\beta$-catenin signaling in adipose precursor cells.

\section{Excessive FAM13A expression inhibits early-stage adipocyte differentiation which could be rescued by PPARy agonist}

We further examined whether ectopic expression of FAM13A in adipose precursor cells impact downstream adipogenesis. When cells were cultured to confluence and subjected to adipocyte differentiation, FAM13A overexpression markedly inhibited adipocyte differentiation as evidenced by impaired protein expression of PPAR $\gamma$ and PLIN1 (Fig. 5a), reduced ORO staining (Fig. 5b) and diminished intracellular TAG content (Fig. 5c) in day 8 (D8) mature adipocytes. mRNA expression of adipocyte marker genes including Ppary, Clebp $\alpha$ and Plinl, were comparable at D0 (2 days after confluence) but significantly 
A

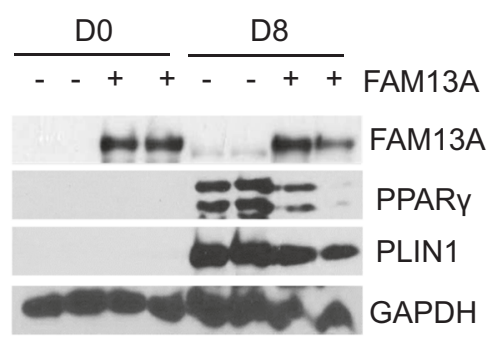

D

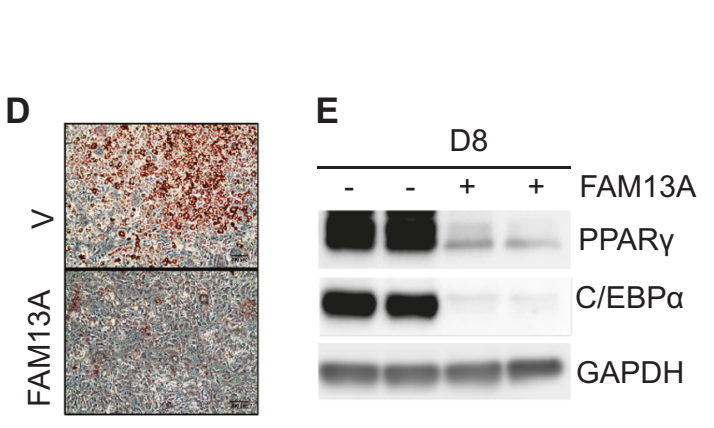

B

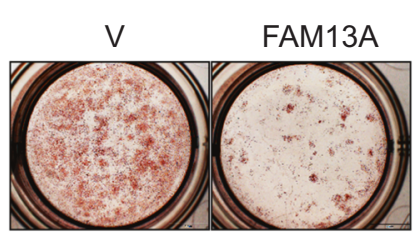

C

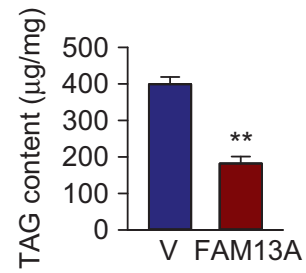

$\mathbf{F}$

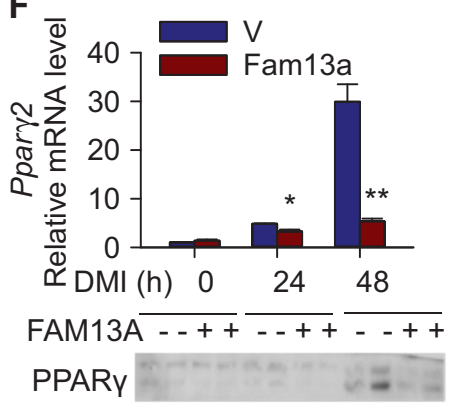

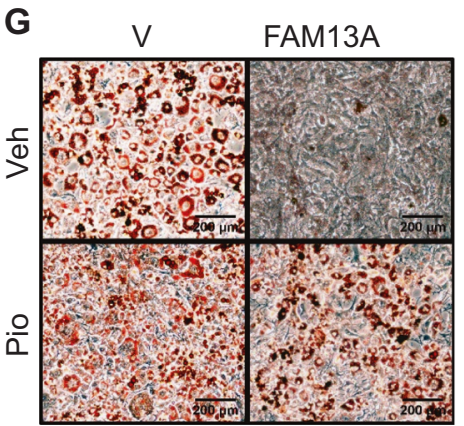

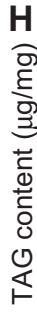

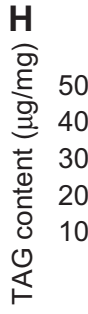

Fig. 5 Excessive expression of FAM13A inhibits adipocyte differentiation. a Same number of cells were initially plated and cultured to confluence for DMI induced differentiation. Western blot at 2 days after confluence (D0) and 8 days (D8) after DMI induction. b, c ORO staining (b) and (c) intracellular TAG content in D8 vector and FAM13A-OE adipocytes. d, e 25\% more FAM13A-OE cells were plated to normalize cell numbers at confluence for differentiation. $\mathbf{d}, \mathbf{e}$

repressed at D8, whereas the preadipocyte marker gene Prefl was upregulated about 4-fold in D8 FAM13A-OE mature adipocytes (Fig. S3a-d, respectively). Notably, the level of lentiviral-expressed FAM13A at D0 was about 10 times higher than the endogenous level of FAM13A in D8 mature adipocytes (Fig. 5a). These data suggest an antiadipogenic effect of FAM13A when overexpressed at super-physiological level. Growth arrest at confluence appears to be a prerequisite for subsequent differentiation [23]. To analyze whether the anti-adipogenic effect of FAM13A is due to reduced survival rate in preadipocytes which may cause uneven confluent status before subjecting to adipocyte differentiation, we plated $25 \%$ more FAM13AOE preadipocytes to ensure both groups reach confluence simultaneously. However, FAM13A-OE preadipocytes were again unable to differentiate into adipocytes based on reduced ORO staining (Fig. 5d) and blunted expression of adipocyte marker proteins (Fig. 5e). Mitotic clonal expansion (MCE) during the first 2 days of differentiation is a prerequisite for terminal adipocyte differentiation [24]. Considering the effect of FAM13A overexpression on preadipocyte survival, we also analyzed MCE and found similar expansion in cell numbers between Vector and FAM13A-OE cells within $48 \mathrm{~h}$ of adipogenesis (Fig. S3e), suggesting MCE was not the direct cause of impaired differentiation in FAM13A-OE cells. Further examination of FAM13A-OE cells identified blocked upregulation of the master adipogenic transcription factor PPAR $\gamma$ as early as $24 \mathrm{~h}$ after DMI stimulation (Fig. 5f). Addition of pioglitazone together with DMI for the first 4 days of differentiation could partially rescue adipogenic deficiency in FAM13AOE cells by restoring TAG contents (Fig. 5g, h) and adipocyte marker protein expression (Fig. 5i). Thus, FAM13A overexpression in adipose precursor cells not only impairs 
survival but also interferes with the normal transcriptional cascade of adipogenesis during fat cell differentiation.

\section{Discussion}

Previous study has identified Fam13a as one of the top ten genes that are dramatically downregulated in adipose tissue of diet-induced obese animals [25]. In this study, we further confirmed this finding in both genetic and diet-induced obese animal models. We also identified Fam13a as an adipose tissue abundant gene highly induced by fasting (dexamethasone) and suppressed by refeeding (insulin), which may partially explain the remarkable downregulation of Fam13a in hyperphagic obese animals. Downregulation of Fam13a in obese adipose tissue could also be attributed to increased adipose inflammation as IL-1 $\beta$ has been previously shown to suppress Fam13a expression [26].

Our study not only identified a striking upregulation of FAM13A during human and murine adipogenesis, but also provided the first evidence for FAM13A as a downstream target of PPAR $\gamma$ during adipocyte differentiation (Fig. 2). Indeed, murine Fam13a promoter contains a potential PPRE binding site (data not shown). Despite its upregulation by PPAR $\gamma$ during adipogenesis, loss of FAM13A exerts no effect on fat cell differentiation (Fig. S1). It also displayed minimal effect on fat distribution under both chow and high fat diet (Fig. 3). As the master regulator of adipogenesis, PPAR $\gamma$ regulates the expression of numerous genes which collectively control adipogenesis. Knockout of PPAR $\gamma$ target genes individually may not always cause detectable phenotypes. While ablation of FAM13A alone is insufficient for causing an adipose development phenotype, we cannot completely rule out the possibility that forced expression of FAM13A in preadipocytes may play a role during adipogenesis (Fig. 5). Clearly, future studies are still needed to fully understand roles of FAM13A in adipose development.

While our manuscript was in the final stage of preparation, Wardhana DA et al. reported similar regulation of Fam13a during adipogenesis. They also found no changes in adiposity in their Fam13a $a^{-1-}$ mice under both chow and high fat diets [27]. However, their $\mathrm{Faml}_{3} \mathrm{a}^{-/-}$mice were insulin resistant and glucose intolerant due to adipose IRS1 downregulation and ATGL upregulation [27]. In our study, we found the expression of IRS1 and ATGL was similar

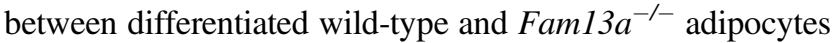
(Fig. S1c) or eWAT and sWAT of normal chow diet (NCD)fed wild type and Fam $13 a^{-/-}$mice (Fig. S2d-g). Consistently, Fam $13 a^{-/-}$adipocytes or NCD-fed Fam13a $a^{-1-}$ mice showed neither differences in response to insulin (Fig. S1d and Fig. 3g, respectively), nor changes in basal and $\beta 3$-adrenergic receptor agonist (CL316,243)-stimulated lipolysis as compared to their respective control groups (data not shown). There was a tendency toward lower IRS1 expression in eWAT and sWAT of Fam $13 a^{-1-}$ mice after HFD feeding (Fig. S2d-g), which did not result in worsened insulin sensitivity and glucose tolerance (Fig. 3i-j). It is not clear whether these discrepancies could be attributed to the differences in diet fat contents or the genetic backgrounds of

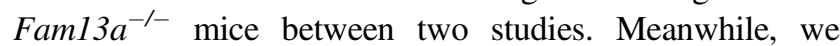
found no changes in SIRT1 protein and Cptl $\alpha$ mRNA expression in either Fam13a-deficient or overexpressed adipose precursor or differentiated adipocytes (Figs. S1e, S3f and S4d, respectively), suggesting FAM13A is dispensable for fatty acid oxidation in adipose depots. Further studies will be required to rigorously test whether FAM13A deficiency affects other adipocyte function in response to metabolic stresses yet to be characterized.

The WHRadjBMI-associated rs9991328 SNP in FAM13A is different from all other SNPs linked to human chronic lung diseases [28]. While COPD-linked SNPs cause higher FAM13A protein expression in lung [13], whether the rs9991328 SNP is linked to abnormal FAM13A expression in human adipose tissue is completely unknown. Importantly, a positive association of FAM13A expression with lung tumor cell proliferation and survival was recently reported [29]; and FAM13A gene expression was also found to exert a stimulatory effect on human adipose hyperplasia [6]. While these findings clearly indicate an important role of FAM13A in regulating tumor or (pre) adipose cell numbers, they seem to be opposite to our finding which suggests a negative role of FAM13A in regulating the survival of adipose precursors. The relationship between FAM13A expression and adipose hyperplasia in humans awaits further study. Notably, FAM13A overexpression in 3T3-L1 preadipocytes reduced total $\beta$-catenin protein expression, confirming its role in promoting $\beta$-catenin degradation [13]. The canonical Wnt pathway has been shown to play an important anti-apoptotic role and is essential for the survival of adipose precursors [22]. Hence, it is conceivable to observe an increased apoptosis in our FAM13A-OE 3T3-L1 preadipocytes (Fig. 4g, h). FAM13A has been shown to affect recruitment of PP2A which modulates GSK3 $\beta$ phosphorylation thus $\beta$-catenin proteasome degradation [13]. Yet, we failed to identify difference in GSK3 $\beta$ phosphorylation in FAM13A-OE preadipocytes (Fig. 4b), suggesting the presence of other regulatory pathways. Nevertheless, our data underscore the important role of FAM13A in regulating adipose precursor cell numbers potentially through mitigating $\beta$-catenin signaling.

$\mathrm{Wnt} / \beta$-catenin pathway represents a major axis upon which various signals converge to influence PPAR $\gamma$ activation and preadipocyte differentiation [30]. Several studies suggest inhibition of $\mathrm{Wnt} / \beta$-catenin signaling in preadipocytes stimulates differentiation [31-33]. Surprisingly, 
despite reduced $\beta$-catenin signaling, FAM13A overexpression did not promote adipogenesis. Instead, it blocked early-stage adipogenesis through blunting PPAR $\gamma$ upregulation, suggesting the presence of a $\beta$-cateninindependent pathway that plays a more dominant role in regulating adipogenesis. Notably, the overexpressed $117 \mathrm{kD}$ isoform of FAM13A contains a Ras homologous GTPaseactivating protein (RhoGAP) domain which potentially mediates Rho GTPase signaling [26, 29], an important pathway that regulates PPAR $\gamma$ and adipogenesis [34]. Future study is needed to dissect whether FAM13A mediates RhoGTPase signaling thus adipogenesis.

The physiologic and/or clinical relevance of FAM13A overexpression in regulating adipose precursor cell survival and adipogenesis is not clear at this point. It is worth to mention that adipose-specific Fam13a-Tg mice lack changes in adipose tissue mass [27], inconsistent with the antiadipogenic role of FAM13A. This discrepancy could result from the fact that FAM13A overexpression is relatively limited to mature adipocytes not adipose precursors in aP2promoter driven Fam $13 a-\mathrm{Tg}$ mice. Thus, animal model in which FAM13A is overexpressed specifically in adipose precursor cells is desirable. Comparison of FAM13A expression and the survival of preadipocytes from adipose tissue of lean and obese humans (carrying rs9991328 SNP at FAM13A) would be ideal. Nevertheless, our data implicate the importance of maintaining temporal expression of Faml3a gene at correct stages of adipose development.

In conclusion, our data pinpoint a dispensable role of FAM13A in adipogenesis and argue against FAM13A as a positive regulator of adipose insulin sensitivity. But forced expression of FAM13A in adipose precursor cells modulates cell survival and early adipogenesis. Hence, our results provide insights to further elucidate mechanisms through which FAM13A contributes to adipose biology.

Acknowledgements We thank James Mintz at Vascular Biology Center at Augusta University for his technical help on NMR analysis of body composition. This work was supported by National Heart, Lung and Blood Institute at National Institute of Health (1R01HL132182-01 [WC]), American Heart Association Grant-in-aid (16GRNT30680004 [WC]) and National Institutes of Health (R01GM111816 [JY]).

\section{Compliance with ethical standards}

Conflict of interest The authors declare that they have no conflict of interest.

Open Access This article is licensed under a Creative Commons Attribution 4.0 International License, which permits use, sharing, adaptation, distribution and reproduction in any medium or format, as long as you give appropriate credit to the original author(s) and the source, provide a link to the Creative Commons license, and indicate if changes were made. The images or other third party material in this article are included in the article's Creative Commons license, unless indicated otherwise in a credit line to the material. If material is not included in the article's Creative Commons license and your intended use is not permitted by statutory regulation or exceeds the permitted use, you will need to obtain permission directly from the copyright holder. To view a copy of this license, visit http://creativecommons. org/licenses/by/4.0/.

\section{References}

1. Maes HH, Neale MC, Eaves LJ. Genetic and environmental factors in relative body weight and human adiposity. Behav Genet. 1997;27:325-51.

2. Visscher PM, Brown MA, McCarthy MI, Yang J. Five years of GWAS discovery. Am J Hum Genet. 2012;90:7-24.

3. Locke AE, Kahali B, Berndt SI, Justice AE, Pers TH, Day FR, et al. Genetic studies of body mass index yield new insights for obesity biology. Nature. 2015;518:197-206.

4. Shungin D, Winkler TW, Croteau-Chonka DC, Ferreira T, Locke AE, Magi R, et al. New genetic loci link adipose and insulin biology to body fat distribution. Nature. 2015;518:187-96.

5. Fagerberg L, Hallstrom BM, Oksvold P, Kampf C, Djureinovic D, Odeberg J, et al. Analysis of the human tissue-specific expression by genome-wide integration of transcriptomics and antibodybased proteomics. Mol Cell Proteom. 2014;13:397-406.

6. Dahlman I, Ryden M, Brodin D, Grallert H, Strawbridge RJ, Arner P. Numerous genes in loci associated with body fat distribution are linked to adipose function. Diabetes. 2016;65:433-7.

7. Cho MH, Boutaoui N, Klanderman BJ, Sylvia JS, Ziniti JP, Hersh $\mathrm{CP}$, et al. Variants in FAM13A are associated with chronic obstructive pulmonary disease. Nat Genet. 2010;42:200-2.

8. Hancock DB, Eijgelsheim M, Wilk JB, Gharib SA, Loehr LR, Marciante KD, et al. Meta-analyses of genome-wide association studies identify multiple loci associated with pulmonary function. Nat Genet. 2010;42:45-52.

9. Pillai SG, Kong X, Edwards LD, Cho MH, Anderson WH, Coxson HO, et al. Loci identified by genome-wide association studies influence different disease-related phenotypes in chronic obstructive pulmonary disease. Am J Respir Crit Care Med. 2010;182:1498-505.

10. Li X, Howard TD, Moore WC, Ampleford EJ, Li H, Busse WW, et al. Importance of hedgehog interacting protein and other lung function genes in asthma. J Allergy Clin Immunol. 2011;127: 1457-65.

11. Young RP, Hopkins RJ, Hay BA, Whittington CF, Epton MJ, Gamble GD. FAM13A locus in COPD is independently associated with lung cancer-evidence of a molecular genetic link between COPD and lung cancer. Appl Clin Genet. 2011;4:1-10.

12. Fingerlin TE, Murphy E, Zhang W, Peljto AL, Brown KK, Steele MP, et al. Genome-wide association study identifies multiple susceptibility loci for pulmonary fibrosis. Nat Genet. 2013;45: 613-20.

13. Jiang Z, Lao T, Qiu W, Polverino F, Gupta K, Guo F, et al. A chronic obstructive pulmonary disease susceptibility gene, FAM13A, regulates protein stability of beta-catenin. Am J Respir Crit Care Med. 2016;194:185-97.

14. Jin Z, Chung JW, Mei W, Strack S, He C, Lau GW, et al. Regulation of nuclear-cytoplasmic shuttling and function of family with sequence similarity 13, member A (Fam13a), by B56containing PP2As and Akt. Mol Biol Cell. 2015;26:1160-73.

15. Jiang Z, Knudsen NH, Wang G, Qiu W, Naing ZZC, Bai Y, et al. Genetic control of fatty acid beta-oxidation in chronic obstructive pulmonary disease. Am J Respir Cell Mol Biol. 2017;56:738-48.

16. Lei X, Callaway M, Zhou H, Yang Y, Chen W. Obesity associated Lyplall gene is regulated in diet induced obesity but not required 
for adipocyte differentiation. Mol Cell Endocrinol. 2015;411: 207-13.

17. Chen W, Chang B, Saha P, Hartig SM, Li L, Reddy VT, et al. Berardinelli-seip congenital lipodystrophy 2/seipin is a cellautonomous regulator of lipolysis essential for adipocyte differentiation. Mol Cell Biol. 2012;32:1099-111.

18. Chen W, Yechoor VK, Chang BH, Li MV, March KL, Chan L. The human lipodystrophy gene product Berardinelli-Seip congenital lipodystrophy $2 /$ seipin plays a key role in adipocyte differentiation. Endocrinology. 2009;150:4552-61.

19. Zhou H, Lei X, Benson T, Mintz J, Xu X, Harris RB, et al. Berardinelli-Seip congenital lipodystrophy 2 regulates adipocyte lipolysis, browning, and energy balance in adult animals. J Lipid Res. 2015;56:1912-25.

20. Villena JA, Roy S, Sarkadi-Nagy E, Kim KH SulHS. Desnutrin, an adipocyte gene encoding a novel patatin domain-containing protein, is induced by fasting and glucocorticoids: ectopic expression of desnutrin increases triglyceride hydrolysis. J Biol Chem. 2004:279:47066-75.

21. Waki H, Park KW, Mitro N, Pei L, Damoiseaux R, Wilpitz DC, et al. The small molecule harmine is an antidiabetic cell-typespecific regulator of PPARgamma expression. Cell Metab. 2007; 5:357-70.

22. Longo KA, Kennell JA, Ochocinska MJ, Ross SE, Wright WS, MacDougald OA. Wnt signaling protects 3T3-L1 preadipocytes from apoptosis through induction of insulin-like growth factors. J Biol Chem. 2002;277:38239-44.

23. Cornelius P, MacDougald OA, Lane MD. Regulation of adipocyte development. Annu Rev Nutr. 1994;14:99-129.

24. Tang QQ, Otto TC, Lane MD. Mitotic clonal expansion: a synchronous process required for adipogenesis. Proc Natl Acad Sci USA. 2003;100:44-9.

25. Choi MS, Kim YJ, Kwon EY, Ryoo JY, Kim SR, Jung UJ. Highfat diet decreases energy expenditure and expression of genes controlling lipid metabolism, mitochondrial function and skeletal system development in the adipose tissue, along with increased expression of extracellular matrix remodelling- and inflammationrelated genes. Br J Nutr. 2015;113:867-77.

26. Corvol H, Rousselet N, Thompson KE, Berdah L, Cottin G, Foussigniere $\mathrm{T}$ et al. FAM13A is a modifier gene of cystic fibrosis lung phenotype regulating rhoa activity, actin cytoskeleton dynamics and epithelial-mesenchymal transition. J Cyst Fibros. 2018;17:190-203.

27. Wardhana DA, Ikeda K, Barinda AJ, Nugroho DB, Qurania KR, Yagi K, et al. Family with sequence similarity 13, member A modulates adipocyte insulin signaling and preserves systemic metabolic homeostasis. Proc Natl Acad Sci USA. 2018;115: 1529-34.

28. Corvol H, Hodges CA, Drumm ML, Guillot L. Moving beyond genetics: is FAM13A a major biological contributor in lung physiology and chronic lung diseases? J Med Genet. 2014;51: 646-9.

29. Eisenhut F, Heim L, Trump S, Mittler S, Sopel N, Andreev K, et al. FAM13A is associated with non-small cell lung cancer (NSCLC) progression and controls tumor cell proliferation and survival. Oncoimmunology. 2017;6:e1256526.

30. Prestwich TC, Macdougald OA. Wnt/beta-catenin signaling in adipogenesis and metabolism. Curr Opin Cell Biol. 2007;19: 612-7.

31. Bennett CN, Ross SE, Longo KA, Bajnok L, Hemati N, Johnson $\mathrm{KW}$, et al. Regulation of Wnt signaling during adipogenesis. J Biol Chem. 2002;277:30998-1004.

32. Li FQ, Singh AM, Mofunanya A, Love D, Terada N, Moon RT, et al. Chibby promotes adipocyte differentiation through inhibition of beta-catenin signaling. Mol Cell Biol. 2007;27: 4347-54.

33. Ross SE, Hemati N, Longo KA, Bennett CN, Lucas PC, Erickson $\mathrm{RL}$, et al. Inhibition of adipogenesis by Wnt signaling. Science. 2000;289:950-3.

34. Sordella R, Jiang W, Chen GC, Curto M, Settleman J. Modulation of Rho GTPase signaling regulates a switch between adipogenesis and myogenesis. Cell. 2003;113:147-58. 